\title{
Влияние электрон-фононного взаимодействия на проводимость и работу выхода эпитаксиального графена
}

\author{
(C) С.Ю. Давыдов
}

Физико-технический институт им. А.Ф. Иоффре Российской академии наук, 194021 Санкт-Петербург, Россия

Санкт-Петербургский национальный исследовательский университет инфрормационных технологий, механики и оптики, 197101 Санкт-Петербург, Россия

E-mail: Sergei_Davydov@mail.ru

(Получена 19 сентября 2017 г. Принята к печати 24 октября 2017 г.)

\begin{abstract}
В рамках кластерной модели эпитаксиального графена получено условие наведенного электрон-фононным взаимодействием графен-подложка и внешним электрическим полем скачкообразного изменения заряда адатомов углерода. Такой переход заряда приводит к соответствующим скачкам статической проводимости и работы выхода эпитаксиального графена. Численные оценки приведены для случая слабой связи квазисвободного графена $c$ металлической и полупроводниковой подложками.
\end{abstract}

DOI: $10.21883 /$ FTP.2018.07.46052.8731

\section{1. Введение}

Переход заряда между адсорбированным атомом и подложкой приводит к двум эффектам [1], первым из которых является изменение работы выхода системы $\Delta \varphi$. Второй эффект - изменение поверхностной проводимости подложки $\Delta G$. Причины этого эффекта двояки. Во-первых, изменяется поверхностная концентрация носителей $\Delta N$; во-вторых, адчастицы представляют собой дополнительные центры рассеяния, что изменяет поверхностную подвижность носителей $\mu$. Начало одновременных измерений поверхностной проводимости и работы выхода положили экспериментальные работы [2,3], посвященные адсорбции газовых молекул на оксидных полупроводниках $\left(\mathrm{H} / \mathrm{ZnO}, \mathrm{O}_{2} / \mathrm{ZnO}\right.$ и $\left.\mathrm{O}_{2} / \mathrm{TiO}_{2}\right)$, доказавшие прямую пропорциональность между значениями $\Delta G(\Theta)$ и $\Delta \phi(\Theta)$, где $\Theta=N_{a} / N_{M L}-$ степень покрытия подложки адчастицами, $N_{a}\left(N_{M L}\right)$ - концентрация адчастиц в слое (монослое).

В работах $[4,5]$ было показано, что изменение концентрации носителей $\Delta N$ равно

$$
\Delta N(\Theta)=\Theta Z(\Theta) N_{M L}
$$

где $Z(\Theta)$ - заряд адатома в слое. С другой стороны, из теории адсорбции известно [1], что изменение работы выхода адсорбционной системы $\Delta \phi$ равно

$$
\Delta \phi(\Theta)=-\Phi \Theta Z_{a}(\Theta),
$$

где $\Phi=4 \pi e^{2} N_{M L} d, d-$ длина адсорбционной связи, $e-$ заряд позитрона. Таким образом, в предположении $|\Delta n| / n \gg|\Delta \mu| / \mu$ получаем следующее отношение $[1,4,5]$ :

$$
\eta \equiv\left|\frac{\Delta G(\Theta)}{\Delta \phi(\Theta)}\right|=\frac{\mu}{4 \pi e d}
$$

Из выражения (3) вытекает отсутствие явной зависимости отношения $\eta$ от степени покрытия $\Theta$. Более того, сопоставление результатов расчета с экспериментом показало достаточно слабую зависимость подвижности электронов $\mu$ от $\Theta$.

Соотношения (1)-(3) в дальнейшем использовались нами [6] для анализа экспериментальных результатов работ [7-10] по адсорбции молекул $\mathrm{NO}_{2}, \mathrm{NH}_{3}$, паров воды, ацетона и метанола $\mathrm{NO}_{2}, \mathrm{NH}_{3}$, паров воды, ацетона и метанола на углеродных наноструктурах. Было продемонстрировано, что переход заряда является главным фактором изменений $\Delta G(\Theta)$ и $\Delta \phi(\Theta)$, наведенных адсорбцией, а зависимостью $\mu(\Theta)$ можно пренебречь.

В настоящей работе формулы (1)-(3) будут применены к эпитаксиальному графену (ЭГ). При этом будет учитываться электрон-фононное взаимодействие листа графена с субстратом, которое, как будет показано, при определенных условиях существенно влияет на переход заряда между графеном и подложкой [11].

\section{2. Адатом углерода}

В соответствии с адсорбционным подходом к задаче об ЭГ [1] начнем с рассмотрения одиночного адатома углерода с энергетическим уровнем $E$ и массой $M$, колеблющегося в перпендикулярном к поверхности субстрата направлении $Z$ с частотой $\omega_{0}$. Не приводя выкладок работы $[11],{ }^{1}$ сразу запишем соответствующую функцию Грина $g(\varepsilon)$, где $\varepsilon$ - энергетическая переменная, в виде

$$
g(\varepsilon)=(\varepsilon-\bar{\varepsilon}-\Lambda(\varepsilon)+i \Gamma(\varepsilon))^{-1},
$$

где

$$
\bar{\varepsilon}=E-\lambda \bar{n}, \quad \lambda=w^{2} / M \omega_{0}^{2} .
$$

\footnotetext{
${ }^{1}$ Отметим, что в уравнении (2) в [11] допущена опечатка. Второе слагаемое должно иметь вид: $M \omega_{0}^{2}\left(z-z_{0}\right)^{2} / 2$.
} 
Здесь $\lambda$ - энергия электрон-фононного взаимодействия, $\bar{n}$ - число заполнения квазиуровня изолированного адатома; функции уширения и сдвига квазиуровня адатома равны соответственно

$$
\begin{aligned}
\Gamma(\varepsilon) & =\pi V^{2} \rho_{\mathrm{sub}}(\varepsilon), \quad \Lambda(\varepsilon) \\
& =V^{2} P \int_{-\infty}^{\infty} d \varepsilon^{\prime} \rho_{\mathrm{sub}}\left(\varepsilon^{\prime}\right) /\left(\varepsilon-\varepsilon^{\prime}\right),
\end{aligned}
$$

где $\rho_{\mathrm{sub}}(\varepsilon)$ - плотность состояний подложки, $V$ матричный элемент взаимодействия адатом-подложка, $P$ - символ главного значения. ${ }^{2}$

Для дальнейшего анализа нужно выбрать модель плотности состояний субстрата $\rho_{\text {sub }}(\varepsilon)$. Полагая для металлической подложи $\rho_{\text {sub }}(\varepsilon)=\rho_{m}=$ const, получим $\Gamma(\varepsilon)=\Gamma_{m}=\pi \rho_{m} V^{2}=$ const и $\Lambda(\varepsilon)=0 \quad$ [1,11-13]. Тогда легко показать, что при нулевой температуре $\bar{n}=2 \pi^{-1} \operatorname{arccot}\left[\left(\bar{\varepsilon}-\varepsilon_{F}\right) / \Gamma_{m}\right]$. Здесь, в отличие от [11], мы рассматриваем как переход заряда с адатома на подложку, так и переход с подложки на адатом. Полагая $\varepsilon_{F}=0$, $e_{m}=E / \Gamma_{m}$ и $\xi=\lambda / \Gamma_{m}$, перепишем уравнение (2) в виде

$$
\cot (\pi \bar{n} / 2)=e_{m}-\zeta_{m} \bar{n} .
$$

При $\xi_{m}<\pi / 2$ имеется только одно решение уравнения (7), при $\xi_{m}>\pi / 2$ таких решений три, причем только два из них (с максимальным и минимальным значениями $\bar{n})$ отвечают устойчивым положениям равновесия адатома.

Сделаем теперь некоторые численные оценки. Для $\mathrm{Rh}, \mathrm{Ir}$ и $\mathrm{Pt}$ ширины $d$-зон $W_{d}$ равны соответственно $6.89,8.71$ и 7.00 эВ [14], так что средние значения $W_{d} \approx 7.5$ эВ и $\rho_{m}=10 / W_{d} \approx 1.3$ эВ ${ }^{-1}$. Анализ экспериментальных данных привел к выводу, что расстояние между слоями графита и подложкой $\operatorname{Pt}(111)$ равно $3.70 \AA[15]$, а между графеновым листом и подложкой $\operatorname{Ir}(111)-3.80 \AA[16]^{3}$ Таким значениям $d$ отвечает ван-дер-ваальсово взаимодействие, характерное для квазисвободного графена. Воспользуемся потенциалом Леннарда-Джонса $V_{L J}(d)=4 D\left[(\bar{\sigma} / d)^{12}-(\bar{\sigma} / d)^{6}\right]$, где равновесное значение $d$ равно $\sqrt[6]{2} \bar{\sigma}$ и $D-$ величина энергии связи. Деформационную константу $w$ определим как производную по $d$ от ван-дер-ваальсова притяжения, что дает $w=12 D / d$. Так как при этом силовая константа $k_{0}=72 D / d^{2}$, получаем $\lambda=2 D$. За длину адсорбционной связи $d$ примем среднее значение постоянных решетки $\mathrm{Pt}$, Ir и $\mathrm{Rh}$, равное $3.85 \AA$, откуда получим $w \approx 3 D$ эВ $/ \AA, k_{0} \approx 5 D$ эВ $/ \AA^{2}$, где $D$ выражено в эВ. Для ван-дер-ваальсовой связи равновесное значение $V=-D$, так что $\Gamma_{m} \approx 4 D^{2}$, где $D$ выражено в эВ. Так как по порядку величины $D \sim 0.1$ эВ, приходим к выводу, что $\Gamma_{m} \ll 1$ эВ, а

\footnotetext{
2 Здесь и в дальнейшем некоторые обозначения отличаются от использованных в работе [11].

3 Эти расстояния весьма близки к постоянным решетки платины и иридия, равным соответственно 3.92 и $3.84 \AA$ [17].
}

$\zeta_{m} \approx(2 D)^{-1}>\pi$. Следовательно, в случае квазисвободного ЭГ на металле возможны два значения чисел заполнения $\bar{n}_{\min }$ и $\bar{n}_{\max }$.

Перейдем теперь к полупроводниковой подложке, задав ее плотность состояний в виде $\rho_{\text {sub }}(\varepsilon)=\rho_{s c}=$ const при $|\varepsilon|>E_{g} / 2 \quad$ и $\rho_{\text {sub }}(\varepsilon)=0$ при $|\varepsilon| \leq E_{g} / 2$, где $E_{g}$ - ширина запрещенной зоны субстрата [18], за нуль энергии принят центр запрещенной зоны. Тогда $\Gamma(\varepsilon)=\Gamma_{s c}=\pi \rho_{s c} V^{2}=$ const, если $|\varepsilon|>E_{g} / 2$, и $\Gamma(\varepsilon)=0$, если $|\varepsilon| \leq E_{g} / 2$, и $\Lambda(\varepsilon)=\left(\Gamma_{s c} / \pi\right) \ln \left|\left(\varepsilon-E_{g} / 2\right) /\left(\varepsilon+E_{g} / 2\right)\right|$.

Обобщая результаты работы [19] на рассматриваемую задачу и считая температуру нулевой, получим вклад валентной зоны в число заполнения $\bar{n}_{v}=\pi^{-1}$ $\times \operatorname{arccot}\left[\left(\bar{\varepsilon}+R-\varepsilon_{F}\right) / \Gamma_{s c}\right]$, где $R=\sqrt{\left(E_{g} / 2\right)^{2}+\Gamma_{s c} E_{g} / \pi}$. Локальный вклад в число заполнения $\bar{n}_{l}=$ $=\left(1+\Gamma_{s c} E_{g} /\left[\left(E_{g} / 2\right)^{2}-\varepsilon_{l}^{2}\right]\right)^{-1}$, где энергия локального уровня $\varepsilon_{l}$ является корнем уравнения $\varepsilon-\bar{\varepsilon}-\Lambda(\varepsilon)=0$ в области $|\varepsilon| \leq E_{g} / 2$. Как показано в [19], при $\bar{\varepsilon}<-E_{g} / 2$ и $\bar{\varepsilon}>\varepsilon_{F}=0$ вкладом локальных состояний можно пренебречь и положить $\bar{n} \approx \bar{n}_{v}$. В этих случаях вновь приходим к уравнению (4), где, однако, нужно заменить $e_{m}$ и $\xi_{m}$ на $e_{s c}=(E+R) / \Gamma_{s c}$ и $\xi_{s c}$.

По данным эксперимента $d \approx 3 \AA$ [20], что характерно для ван-дер-ваальсова взаимодействия, т. е. для квазисвободного ЭГ. Так как для тетраэдрических полупроводников значения ширины валентной зоны порядка 10-12 эВ, получим $\Gamma_{s c} \approx D^{2}$, где $D$ выражено в эВ. Так как $\lambda=2 D$, то $\xi_{s c} \approx 2 / D \gg \pi$. Таким образом, и в случае квазисвободного ЭГ на полупроводнике имеются два значения чисел заполнения $\bar{n}_{\min }$ и $\bar{n}_{\max }$. В дальнейшем там, где материал относится как к ЭГ на металле, так и к ЭГ на полупроводнике, мы будем опускать нижние индексы $m$ и $s c$.

Необходимо отметить, что при $\xi>\pi / 2$ и $|e-\xi|>B(\xi), \quad$ где $\quad B(\xi)=(2 \xi / \pi) \arccos (\pi / 2 \xi)^{1 / 2}$ $-[(2 \xi / \pi)-1]^{1 / 2}$, имеется одно устойчивое положение равновесия адатома. При $|e-\xi|<B(\xi)$ таких устойчивых положений два: если $e>\xi$, то минимуму энергии системы соответствует $\bar{n}_{\min }$, при $e<\xi$ минимуму энергии отвечает $\bar{n}_{\text {max }}$. Значениям $\pm B(\xi)$ отвечают числа заполнения $\bar{n}_{\text {min,max }}^{*}=1 \mp(2 / \pi) \arccos \left[(\pi / 2 \xi)^{1 / 2}\right]$. Если, приложив внешнее электрическое поле, сдвигать уровень $e$, то при $e=\xi>\pi / 2$ происходит скачкообразный переход между состояниями с $\bar{n}_{\min }$ и $\bar{n}_{\max }$, которые, как не трудно показать, являются корнями уравнения $\xi(\bar{n}-1)=\tan [(\pi / 2)(\bar{n}-1)]$. Сказанное представлено рисунком $a$ и $b$. На рисунке $c$ приведены зависимости $B$ и разности $\Delta \bar{n}^{*}=\left(\bar{n}_{\max }^{*}-\bar{n}_{\min }^{*}\right)$, где $\bar{n}_{\max }^{*}$ и $\bar{n}_{\min }^{*}$ отвечают соответственно $-B$ и $B$, от $\xi$.

При малых степенях покрытия $\Theta \ll 1$, когда адатомы можно считать независимыми, величина скачка работы выхода $|\delta \phi(\Theta)|$ при переходе уровня адатома через значение $e=\xi$, согласно (2), будет равна $|\delta \phi(\Theta)|=\Phi \Theta|\delta \bar{Z}(0)|=\Phi \Theta \Delta \bar{n}$, где $\Delta \bar{n}=\left(\bar{n}_{\max }-\bar{n}_{\min }\right)$. 

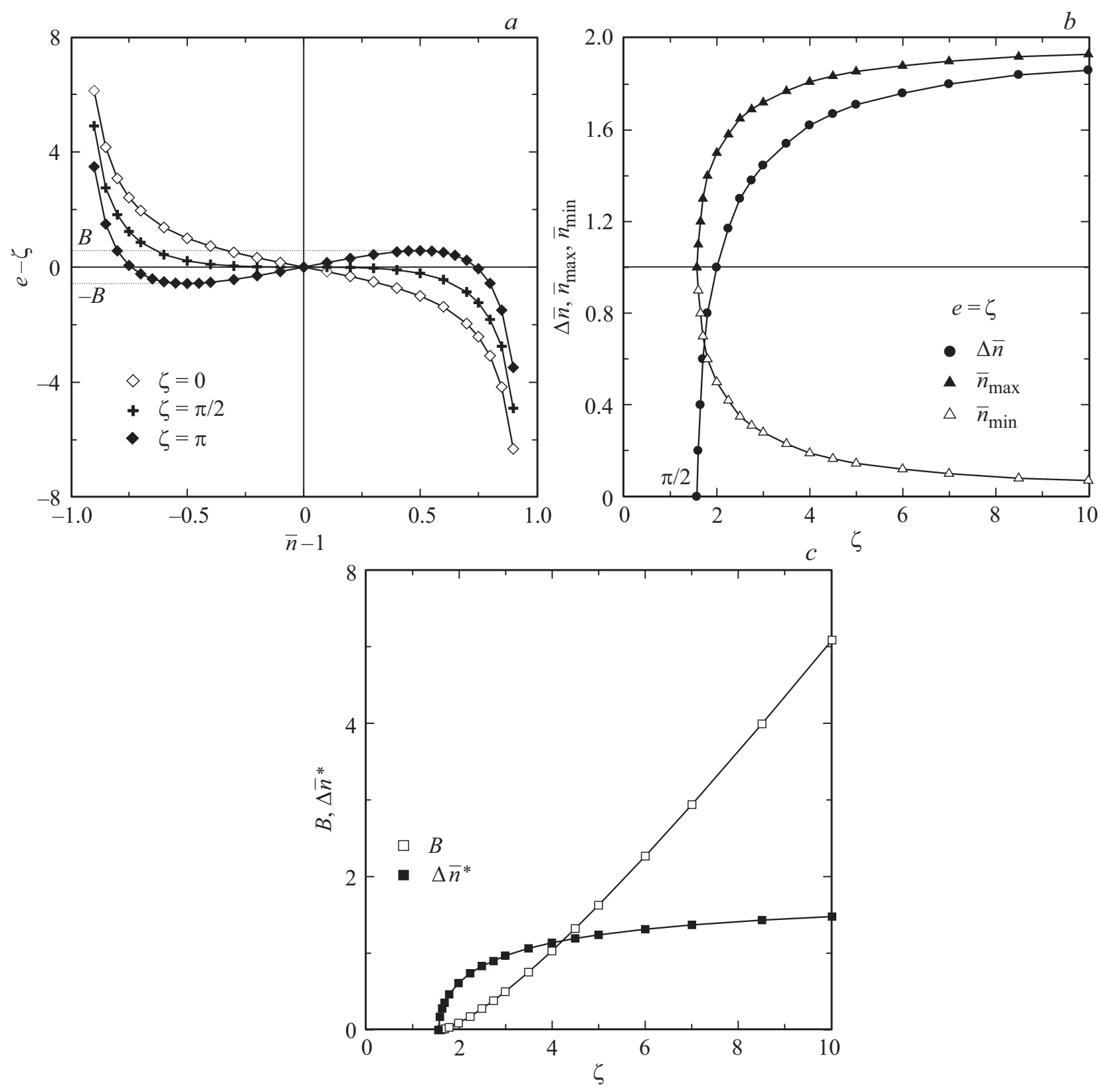

Изолированный адатом углерода. $a-$ зависимости чисел заполнения квазиуровня адатома $\bar{n}$ от его приведенной энергии $e$ в отсутствие $(\xi=0-$ светлые ромбы) и при наличии $(\xi=\pi / 2-$ крестики, $\xi=\pi$ - темные ромбы) электрон-фононной связи адатом-подложка; выражение для параметра $B$ приведено в тексте; $b-$ зависимости максимального $\bar{n}_{\max }$ и минимального $\bar{n}_{\min }$ чисел заполнения и величины перехода заряда $\Delta \bar{n}=\left(\bar{n}_{\max }-\bar{n}_{\min }\right)$ от $\xi=e ; c-$ зависимости приведенной энергии $B$ и разности $\Delta \bar{n}^{*}=\left(\bar{n}_{\max }^{*}-\bar{n}_{\min }^{*}\right)$ от константы электрон-фононной связи $\zeta$. Значения $\bar{n}_{\max }^{*}$ и $\bar{n}_{\min }^{*}$ отвечают соответственно приведенным энергиям $-B$ и $B$.

\section{3. Эпитаксиальный графен: кластерная модель}

Воспользуемся кластерной моделью ЭГ, хорошо зарекомендовавшей себя для описания электрон-электронного [21] и электрон-фононного взаимодействий [22]. Для этого выстроим из шести адатомов углерода кластер, изображенный на рис. 3 работы [21], и включим между адатомами кинетический обмен $t\left(a_{0}^{+} a_{i}+a_{i}^{+} a_{0}\right)$, где $t \sim 3$ эВ - энергия перескока электрона между б. с., $a_{0(i)}^{+}\left(a_{0(i)}-\right.$ оператор рождения (уничтожения) электрона на нулевом $(i$-м) адатоме. Воспользовавшись результатами [21], получим плотность состояний (на один адатом) вида

$$
\rho(\varepsilon)=\frac{\Gamma}{2 \pi}\left(\frac{1}{(\varepsilon-\bar{\varepsilon}-\sqrt{z} t)^{2}+\Gamma^{2}}+\frac{1}{(\varepsilon-\bar{\varepsilon}+\sqrt{z} t)^{2}+\Gamma^{2}}\right) .
$$

Таким образом, плотность состояний кластера представляет собой суперпозицию плотностей состояний двух 
квазиуровней, центрированных при энергиях $\bar{\varepsilon} \mp \sqrt{z} t$, что формально совпадает с результатами двухпримесной задачи Александера и Андерсона [23] и модели эпитаксиального димера [24]. Рассмотренная ситуация аналогична наличию связывающих и антисвязывающих уровней в двухатомной молекуле.

Полагая температуру нулевой, для металлической подложки получим

$$
n=\frac{1}{\pi}\left(\operatorname{arccot} \frac{\bar{\varepsilon}_{m}-\sqrt{z} t-\varepsilon_{F}}{\Gamma_{m}}+\operatorname{arccot} \frac{\bar{\varepsilon}_{m}+\sqrt{z} t-\varepsilon_{F}}{\Gamma_{m}}\right) .
$$

Сделаем некоторые численные оценки. Положим $E=-(I+A) / 2=-6.26$ эВ относительно вакуума, ${ }^{4}$ где для углерода потенциал ионизации $I=11.26$ эВ, а сродство к электрону $A=1.26$ эВ [25]. Тогда $E=-6.26$ эВ. Значения работы выхода различных граней иридия лежат в интервале $5.42-5.76$ эВ, золота - в интервале $5.31-5.47$ эВ. Таким образом, энергия уровня адатома $E \approx-(1.0-1.5)$ эВ относительно $\varepsilon_{F}=0$. Уточним теперь, что вследствие кинетического обмена нижний по энергии квазиуровень смещается на $\sqrt{z} t \sim 5$ эВ вниз, тогда как верхний квазиуровень - на ту же величину вверх. При этом нижний квазиуровень почти заполнен, а верхний квазиуровень почти пуст. Пренебрегая для простоты вторым слагаемым в (9), получим

$$
\cot (\pi n) \approx e_{m}-\tau_{m}-\xi_{m} n,
$$

где $\tau_{m}=\sqrt{z} t / \Gamma_{m}$. Легко показать (см. $\left.[1,11]\right)$, что скачок чисел заполнения адатомов имеет место при $\xi_{m}>\pi$ и $e_{m}-\tau_{m}=\xi_{m}$. Выполнения последнего условия можно добиться, сдвигая внешним электрическим полем квазиуровни адатома вверх. ${ }^{5}$ При этом (9) переходит в уравнение $\tan [\pi(n-1 / 2)]=\xi_{m}(n-1 / 2)$. Полагая $n=1 / 2+v_{m}, \quad$ где $\quad\left|v_{m}\right| \ll 1, \quad$ получаем $\left|v_{m}\right| \approx(1 / \pi) \sqrt{3\left[\left(\xi_{m} / \pi\right)-1\right]}$, что справедливо при $\left[\left(\xi_{m} / \pi\right)-1\right] \ll 1$. При этом скачкообразное уменьшение чисел заполнения равно $2\left|v_{m}\right|$.

Перейдем к оценкам для полупроводниковой подложки. Так, например, для гексагональных и ромбоэдрических политипов карбида кремния $E_{g} \sim 3$ эВ [26]. Того же порядка и уровень сродства к электрону $\chi$ [27]. Следовательно, центр запрещенной зоны, принятый нами за нуль энергии, лежит на $\sim 4.5$ эВ ниже уровня вакуума. Нижний по энергии квазиуровень адатома углерода отвечает энергии $E=-1.76$ эВ, т. е. расположен на 0.26 эВ ниже потолка валентной зоны. С учетом вклада $(-\sqrt{z} t)$ нижний квазиуровень смещается в глубину валентной зоны и является почти заполненным, верхний же квазиуровень находится выше уровня Ферми, совпадающего

\footnotetext{
4 Энергия отталкивания электронов на внешней орбитали есть $U=I-A$. В немагнитном режиме можно положить $E=-I+U / 2[1]$.

${ }^{5}$ Сдвиг квазиуровней адатома эквивалентен смещению точки Дирака ЭГ относительно уровня Ферми подложки. Нужно, однако, иметь в виду, что в точке Дирака плотность состояний ЭГ на металле в нуль никогда не обращается. То же относится и к полупроводниковой подложке, если точка Дирака находится вне запрещенной зоны.
}

с центром запрещенной зоны, и является почти пустым. Имеем, таким образом, рассмотренную выше „металлическую ситуацию“. Тогда выражения (9), (10) справедливы при замене $e_{m}, \xi_{m}, \tau_{m}$ на $\varepsilon_{s c}, \xi_{s c}, \tau_{s c}=\sqrt{z} t / \Gamma_{s c}$. Вновь подавая на ЭГ потенциал, сдвинем нижний квазиуровень адатома так, чтобы выполнялось условие $e_{s c}-\tau_{s c}=\xi_{s c}$, что приведет к уменьшению числа заполнения на величину $2\left|v_{s c}\right|$, где $v_{s c}=n-1 / 2$. Если $\left[\left(\xi_{s c} / \pi\right)-1\right] \ll 1$, то $\left|v_{s c}\right| \approx(1 / \pi) \sqrt{3\left[\left(\xi_{s c} / \pi\right)-1\right]}$.

Мы считаем излишним приводить здесь для кластера рисунки, аналогичные рисунку для изолированного адатома углерода. Действительно, с качественной точки зрения рисунки $a$ и $c$ справедливы и в настоящем случае при замене $e-\xi$ и $\bar{n}-1$ на $e-\xi / 2$ и $n-1 / 2$, где, однако, $B(\xi)=(\xi / \pi) \arccos (\pi / \zeta)^{1 / 2}-[(\xi / \pi)-1]^{1 / 2}$, а значения чисел заполнения в 2 раза меньше; то же относится к рисунку $b$, где $\xi$ нужно заменить на $\xi / 2$ [28].

Величина скачка концентрации носителей в слое графена равна $\delta(N)=2|v| / S_{1}$, где $S_{1}=(3 \sqrt{3} / 4) a^{2}-$ площадь, приходящаяся на один атом листа (монослоя) графена, $a=1.42 \AA-$ расстояние между ближайшими соседями (б.с.) в графене. В то же время изменение чисел заполнения ведет к скачкообразному понижению работы выхода системы на величину $\delta(\phi)=2 \Phi(v)$, где для графена $\Theta=1$. Здесь, однако, встает обычный для теории адсорбции вопрос о некоторой неопределенности оценки значения концентрации $N_{M L}$, входящей в выражение $\Phi=4 \pi e^{2} N_{M L} d[1] .^{6}$ Поэтому проще сравнивать с экспериментом отношение $2\left|\phi_{+}-\phi_{-}\right| /\left(\phi_{+}+\phi_{-}\right)$, где $\phi_{+(-)}$- работа выхода системы после (до) скачка чисел заполнения.

\section{4. Обсуждение результатов и заключение}

Итак, в настоящей работе определено условие для скачкообразного перехода заряда между графеном и подложкой. Такой переход возможен, во-первых, только при достаточно большой константе электрон-фононной связи графен-подложка и, во-вторых, при наличии внешнего электрического поля, позволяющего смещать точку Дирака ЭГ относительно уровня Ферми подложки. При этом ЭГ моделировался кластером из шести адатомов углерода.

Следует подчеркнуть, что использование кластерной модели ЭГ позволяет избежать трудностей, возникающих при вычислении чисел заполнения в рамках популярного в теории графена низкоэнергетического приближения. Это приближение состоит в замене реального электронного спектра свободного графена $\varepsilon(\mathbf{k})$ на линеаризованное выражение вида $\varepsilon_{ \pm}(\mathbf{q})=E+3 t a|\mathbf{q}| / 2$, где $\mathbf{q}=\mathbf{K}-\mathbf{k}$, где $\mathbf{K}=a^{-1}(2 \pi / 3 \sqrt{3}, 2 \pi / 3)-$ волновой вектор точки Дирака, верхний знак относится к зоне

\footnotetext{
6 Экспериментаторы также избегают оценок $N_{M L}$, предпочитая пользоваться временем экспозиции или количеством нанесенного на подложку адсорбата.
} 
проводимости, нижний - к валентной зоне. Таким образом, плотность состояний ЭГ достаточно хорошо описывается лишь вблизи точки Дирака, тогда как для вычисления чисел заполнения нужно знать плотность состояний во всем энергетическом диапазоне.

В настоящей статье мы не учитывали релаксацию листа ЭГ относительно подложки, так как этот вопрос рассматривался в работе [11]. Учет такой релаксации приводит к тому, что длины адсорбционной связи $d$ до и после скачка перехода заряда различаются. Это ведет к различиям параметров $\lambda, \Gamma$ и $\Phi$ задачи. В принципе такие различия учесть не сложно. Эти различия, однако, будут достаточно малы по сравнению с $d \sim 3-4 \AA$, что для сделанных выше оценок несущественно.

В заключение отметим, что экспериментальное обнаружение скачков работы выхода и поверхностной проводимости ЭГ могло бы дать информацию о константе электрон-фононной связи графен-подложка.

\section{Список литературы}

[1] С.Ю. Давыдов. Теория адсорбции: метод модельных гамильтонианов (СПб., Изд-во СПбГЭТУ ЛЭТИ, 2013). Электронный адрес: twirpx.com/file/1596114/

[2] W. Göpel. Prog. Surf. Sci., 20, 9 (1985).

[3] V.E. Henrich, P.A. Cox. The Surface Science of Metal Oxides (Cambridge, University Press, 1994).

[4] Д.Г. Аньчков, С.Ю. Давыдов, С.В. Трошин С.В. Письма ЖТФ, 33 (18), 47 (2007).

[5] Д.Г. Аньчков, С.Ю. Давыдов, С.В. Трошин. Письма ЖТФ, 34 (18), 54 (2008).

[6] С.Ю. Давыдов, О.В. Посредник. ЖТФ, 87, 635 (2017).

[7] M. Qazi, T. Vogt, G. Koley. Appl. Phys. Lett., 91, 233101 (2007).

[8] M. Qazi, T. Vogt, G. Koley. Appl. Phys. Lett., 92, 103120 (2008).

[9] Md.W.K. Nomani, R. Shishir, M. Qazi, D. Diwan, V.B. Shield, M.G. Spencer, G.S. Tompa, N.M. Sbrockey, G. Koley. Sensors Actuators B, 150, 301 (2010).

[10] M. Qazi, J. Liu, M.V.S. Chandrashekhar, G. Koley. J. Appl. Phys., 106, 094901 (2009).

[11] С.Ю. Давыдов. ФТТ, 55, 197 (2013).

[12] P.W. Anderson. Phys. Rev., 124, 41 (1961).

[13] Т. Мория. Спиновые фллктуации в магнетиках с коллективизированными электронами (М., Мир, 1988).

[14] В.Ю. Ирхин, Ю.П. Ирхин. Электронная структура, физические свойства и корреляционные эфбфекты в $d$ u $f$-металлах и их соединениях (Екатеринбург, УрО PAH, 2004).

[15] H. Zi-Pu, D.F. Ogletree, M.A. Van Hove, G.A. Somorjai. Surf. Sci., 180, 433 (1987).

[16] A.T. N'Diaye, S. Bleikamp, P.J. Feibelman, T. Michely. Phys. Rev. Lett., 97, 215501 (2006).

[17] Ч. Киттель. Введение в физику твердого тела (М., Наука, 1978).

[18] F.D.M. Haldane, P.W. Anderson. Phys. Rev. B, 13, 2553 (1976).

[19] С.Ю. Давыдов, С.В. Трошин. ФТТ, 49, 1508 (2007).
[20] J. Borysiuk, J. Sołtys, R. Bozek, J. Piechota, S. Krukowski, W. Strupinski, J. M. Baranowski, R. Stepniewski. Phys. Rev. B, 85, 045426 (2012).

[21] С.Ю. Давыдов. ФТТ, 58, 779 (2016).

[22] С.Ю. Давыдов. ФТП, 52, 238 (2018).

[23] S. Alexander, P.W. Anderson. Phys. Rev., 133, A1594 (1963).

[24] С.Ю. Давыдов. ФТТ, 54, 2193 (2012).

[25] Физические величины. Справочник, под ред. И.С. Григорьева, Е.3. Мейлихова (М., Энергоатомиздат, 1991).

[26] В.И. Гавриленко, А.М. Грехов, Д.В. Корбутяк, В.Г. Литовченко. Оптические свойства полупроводников. Справочник (Киев, Наук. думка, 1987).

[27] С.Ю. Давыдов. ФТП, 41, 718 (2007).

[28] С.Ю. Давыдов. ФТП, 47, 97 (2013).

Редактор Г.А. Оганесян

\section{Effect of electron-phonon interaction on the conductivity and work-function of epitaxial graphene}

\author{
S.Yu. Davydov
}

loffe Institute, 194021 St. Petersburg, Russia

National Research University

of Information Technologies, Mechanics and Optics, 197101 St. Petersburg, Russia

Abstract Within the scope of the cluster model for epitaxial graphene the condition of the carbon adatom's charge jumplike variation induced by the graphene-substrate electron-phonon coupling and external electric field is obtained. Such a charge transfer leads to the corresponding jumps in the epitaxial graphene static conductivity and work-function. Numerical estimations are fulfilled for the quasi-free-standing graphene on metal and semiconductor substrates. 